\title{
Very late recurrences after ablation of AVNRT
}

\author{
Per Insulander ${ }^{1}$ and Mats Jensen-Urstad ${ }^{1}$ \\ ${ }^{1}$ Karolinska University Hospital
}

February 10, 2021

\begin{abstract}
Very late recurrences after ablation of AVNRT have been reported. Age related alterations of nodal tissues caused by fibrous and fatty tissue infiltration and changes of the sympathovagal influence on the AV node, in turn altering AV nodal conduction and refractoriness, could set the stage for a previously not present substrate for AVNRT. Consequently, the occurrence of AVNRT many years after an ablation procedure may perhaps not always implicate a recurrence but instead an arrhythmia caused by a new substrate.
\end{abstract}

Letter to the editor

Very late recurrences after ablation of AVNRT

Per Insulander, MD PhD, Mats Jensen-Urstad, MD PhD

Dept of Cardiology at Karolinska Institutet and Karolinska University Hospital

Corresponding author:

Per Insulander

Dept of Cardiology at Karolinska Institutet

Karolinska University Hospital, M85

S-141 86 Stockholm

Sweden

Tel +46858580000

Fax +46858586710

per.insulander@sll.se

Funding: none

Conflict of interest: none

We read with great interest the recently published article by Chaumont et al. (1) regarding late recurrences in AVNRT after cryoablation. The authors found that among 257 patients with AVNRT successfully ablated with cryo energy, recurrences occurred in 24 subjects during a follow-up of $38 \pm 27$ months. Most of the recurrences occurred between 1- and 6-year follow-up, with one-third recurrences occurring after the 3-year follow-up. Seventeen patients demonstrated the fast-slow or slow-slow variants of AVNRT. In univariate analysis, procedure duration, number of applications, transient PR prolongation or AV block (5 cases), and Kock's triangle anatomical variants were associated with AVNRT recurrences. However, only the last characteristic was independently associated with recurrence. 
Some years ago, we addressed this issue in a larger cohort of patients who underwent cryoablation of AVNRT (2). In all, we followed up 516 patients (mean age 50 years, range 13-89 years, 317 women) for a mean of 7.1 years (range 2-11.5 years). All were ablated with the $6 \mathrm{~mm}$ Freezer-Extra catheter. In this cohort, 24 patients had the fast-slow or slow-slow variants of AVNRT. Furthermore, transient AV block was observed in 45 patients. The anatomic morphology of Kock's triangle was not regularly documented in this series. In all, 54 patients had recurrences.

In contrast to the finding of Chaumont et al., atypical variants of AVNRT were related to recurrence in our series, but transient AV block and energy duration were not. In agreement with Chaumont et al., late recurrences dominated. Of these, 19 occurred later than 1 year, 14 occurred later than 2 years, 8 occurred later than 3 years, and 6 occurred later than 4 years. One patient had recurrence after 9 years.

The issue of very late recurrences, 5-10 years after the index procedure, also reported after RF ablation (3), is interesting.

In our opinion, recurrence of AVNRT due to AV nodal pathway reconduction caused by suboptimal cryo or RF lesions is less likely so many years after the index procedure. We think other explanations should be discussed.

The ablation lesion per se may certainly evolve to constitute a new substrate but would probably manifest itself earlier. We think that age related effects should also be considered. Fibrous and fatty tissue infiltration in cardiac nodal tissues has been described to start as early as in subjects 35-40 years old (4). Furthermore, the sympathovagal influence on the AV node changes with age, partly due to changes in the proportion of beta-adrenergic receptors and to a decrease in vagal influence, in turn altering AV nodal conduction and refractoriness $(5,6)$. Taken together, these age-related changes in AV nodal electrophysiologic conditions could set the stage for a previously not present substrate for AVNRT. It is not uncommon that AVNRT manifest itself for the first time in remarkably high age. This may also be explained by the previously mentioned age-related AV nodal changes.

Consequently, the occurrence of AVNRT many years after an ablation procedure may perhaps not always implicate a recurrence but instead an arrhythmia caused by a new substrate.

Word count $499(\max 500)$

\section{References}

1. Caumont C, Mirolo A, Savouré A, Godin B, et al. Very long-term outcomes after catheter ablation of AVNRT: How does cryoenergy differ from radiofrequency? J Cardiovasc Electrophysiol.2020;32:32153222 .

2. Insulander P, Bastani H, Braunschweig F, Drca N, et al. Cryoablation of atrioventricular nodal reentrant tachycardia: 7-year follow-up in 515 patients-confirmed safety but very late recurrences occur. Europace. 2017;19:1038-1042.

3. Feldman A, Voskoboinik A, Kumar S, Speznce S et al. Predictors of acute and long-term success of slow pathway ablation for atrioventricular nodal reentrant tachycardia: a single center series of 1,419 consecutive patients. Pacing Clin Electrophysiol. 2012;34:927-933

4. Comunoglu C, Comunoglu N, Eren B, Tanrlöver O, et al. Age-related histopathological changes in the cardiac conducting system in the Turkish population: an evaluation of 202 autopsy cases. Folia Morphol.2012;71:178-182.

5. Brodde OE, Michel MC. Adrenergic and muscarinic receptors in the human heart. Pharmacol Rev. 1999;51:651-690.

6. Kuo C-T, Wu J-M, Lin K-H, Young M-L. The effects of aging on AV nodal recovery properties. Pacing Clin Electrophysiol. 2001;24:194-198. 